\title{
Suppurative Parotitis in a Girl: A Case Report From Ahvaz, Iran
}

\author{
Seyed Mohammad Hassan Aletayeb ${ }^{1}$; Ashraf Sepehran ${ }^{1}$; Hazhir Javaherizadeh ${ }^{1, *}$ \\ ${ }^{1}$ Department of Pediatrics, Abouzar Children's Hospital, Faculty of Medicine, Ahvaz Jundishapur University of Medical Sciences, Ahvaz, IR Iran \\ ${ }^{*}$ Corresponding author: Hazhir Javaherizadeh, Department of Pediatrics, Abouzar Children's Hospital, Faculty of Medicine, Ahvaz Jundishapur University of Medical Sciences, Ahvaz, \\ IR Iran. Tel/Fax: +98-6113337681, E-mail: Hazhirja@yahoo.com
}

Received: May 19, 2013; Revised: September 8, 2013; Accepted: September 25, 2013

\begin{abstract}
Introduction: Neonatal parotitis is a rare disease. Neonatal suppurative parotitis commonly presents with facial swelling, irritability, tenderness of parotid region, and with or without fever. Acute neonatal suppurative parotitis is one of the differential diagnoses of facial swelling with a prevalence of 3.8/10'000 of neonatal admission.

Case Presentation: A 32-day-old girl with fever and restlessness was admitted in the hospital. Left facial swelling was found during physical examination. Redness was observed in the face. Prenatal history was normal. Birth weight was $3500 \mathrm{~g}$. Body weight, length, and head circumference were $4300 \mathrm{~g}$ ( 75 th percentile), $52 \mathrm{~cm}$ ( 50 th percentile), and $38 \mathrm{~cm}$ ( 75 th percentile), respectively. She was breastfed. Pulse and respiratory rates were $130 / \mathrm{min}$ and 50/min, respectively. Axillary temperature was $37.8^{\circ} \mathrm{C}$. Head examination revealed normal sized fontanel $(1.5 \times 1.5 \mathrm{~cm})$ without bulging. Eye and ear were normal. Abdominal examination revealed no abnormal findings. Results of urine analysis and culture were normal. Blood urea nitrogen, sodium, potassium, and blood sugar were normal. Blood amylase was $10 \mathrm{U} / \mathrm{L}$. Bilateral multiple reactive lymph node $($ size $=6 \times 10 \mathrm{~mm}$ ) at anterior cervical chain with a left facial swelling was observed in ultrasonography report. Pus was obtained following gentle pressure on Stensen's duct. Staphylococcus aureus was detected in the microscopic and microbiological evaluations.The patient received a seven-day treatment course with vancomycin and amikacin. Neonate was discharged in a good condition.

Conclusions: Acute suppurative parotitis should be suspected in infants with fever, and irritability in pre-auricular region; and should be treated with appropriate antibiotics.
\end{abstract}

Keywords:Parotitis; Staphylococcus aureus; Fever; Stensen's Duct

\section{Introduction}

Neonatal suppurative parotitis is rare (1) and commonly presents with facial swelling, irritability, tenderness of parotid region, and with or without fever. Acute neonatal suppurative parotitis is one of differential diagnoses of facial swelling with a prevalence of 3.8/10,000 of neonatal admission (2). Higher incidence was observed in premature newborns (2). Staphylococcus aureus was the most frequent offending organism, but other Gram-positive, Gram-negative and anaerobic organism may be involved (2). Intravenous antibiotic therapy is recommended for treatment. Surgical treatment is needed in complicated cases such as intra-glandular abscess (3). The current report is regarding a 32 day-old girl infant with suppurative parotitis. Limited published case reports were found from our country after searching Pubmed and Google Scholar with keywords of neonatal parotitis, suppurative parotitis, and infant.

\section{Case Report}

A 32- day-old girl with fever and restlessness was admitted in the hospital. Parents reported fever and restlessness on the day before admission. There was no coryza or conjunctivitis. Left facial swelling was found during physical examination. Left sub-mandibular swelling was noted in physical examinations. Redness was observed in the face. Prenatal history was normal. Postnatal history was normal. Parents were close relatives. Cesarean section was used for the delivery because of breech presentation. Birth weight was $3500 \mathrm{~g}$. Body weight, length, and head circumference were $4300 \mathrm{~g}$ (75 th percentile), $52 \mathrm{~cm}$ (50 th percentile), and $38 \mathrm{~cm}$ (75 th percentile), respectively. She was breastfed. Pulse and respiratory rates were $130 / \mathrm{min}$ and 50/min, respectively. Axillary temperature was $37.8^{\circ} \mathrm{C}$. Head examination revealed normal sized fontanel $(1.5 \times 1.5 \mathrm{~cm})$ without bulging. Facial examination revealed swelling and erythema over the parotid gland. The patient showed irritability during inspection of the area of swelling. Eye and ear were normal. Abdominal examination revealed no abnormal findings.

Chest examination revealed no abnormality. Red blood cell (RBC) and white blood cell (WBC) counts were $3.1 \times$ $10^{12}$ cells/L and $12.6 \times 10^{9} / \mathrm{L}$ (neutrophil: $60 \%$ ), respectively. Erythrocyte sedimentation rate and C-reactive protein were $20 \mathrm{~mm} / \mathrm{L}$ and negative, respectively. Results of urine analysis and culture were normal. Blood urea nitrogen, sodium, potassium, and blood sugar were normal. Blood amylase was $10 \mathrm{U} / \mathrm{L}$. Bilateral multiple reactive lymph

Copyright @ 2014,Ahvaz Jundishapur University of Medical Sciences; Published by Kowsar. This is an open-access article distributed under the terms of the Creative Commons Attribution License, which permits unrestricted use, distribution, and reproduction in any medium, provided the original work is properly cited. 
node $($ size $=6 \times 10 \mathrm{~mm})$ at anterior cervical chain with a left facial swelling was observed in ultrasonography report. Chest roentgenogram was normal. Due to suspected neonatal parotitis, with gentle pressure on Stensen's duct, pus was evacuated and transferred to the laboratory. Microscopic examinations of the smear revealed Gram-positive cocci. Culture showed coagulase positive S. aureus. Patient received vancomycin and amikacin for a seven-day period. She was discharged in a good condition.

\section{Discussion}

Neonatal suppurative parotitis is a rare disease. Since 2004, 16 additional cases were reported (3). Neonatal suppurative parotitis is one of the differential diagnoses of facial swelling which include trauma, lipoma, and adenomas (1). Ascending bacteria from the oral cavity is the commonest source of infection (4). The most common presentation of neonatal parotitis is fever, swelling, and erythema in the pre-auricular area. Our patient had similar presentation as mentioned in the previous published literature. Leuckocytosis above $15 \times 10^{9} / \mathrm{L}$ with the neutrophil predominance was found in $71 \%$ of the cases, and erythrocyte sedimentation rate (ESR) was elevated in $20 \%$ of the cases (5). The patient under study had normal ESR, WBC count was less than $15 \times 10^{9} / \mathrm{L}$, and serum amylase within the normal limit. Insufficient breast feeding, hot weather (6), excessive oral suctioning, naso-gastric tube feeding, and maternal breast abscess are the possible risk factors (7).

Low birth weight, immunosuppression and oral trauma were reported as the risk factors for parotitis (1). About half of the cases had the risk factors (3). The present case had been breastfeed well because of her weight. Therefore, insufficient breast feeding was not a possible risk factor. Oral manipulation by grandmother was reported in the present case. The patient was admitted in winter. Neonatal suppurative parotitis is more common among males, with a rate of 3:1 (2). Of the 16 cases reported since 2004,13 cases were male (3). S. aureus is the most common pathogen implicated in the neonatal parotitis. Escherichia coli, Klebsiella pneumonia, and Pseudomonas aeruginosa were also reported (4). Microscopic and microbiological evaluations showed $S$. aureus which was consistent with most of the reports. The present report found Methicillin Resistant S. aureus (MRSA) as a cause of neonatal suppurative parotitis (8). Most of the cases were unilateral $(2,3)$. The infection may be bilateral (4). The patient had unilateral swelling which was similar to that of similar literature $(2,3)$.

Intravenous vancomycin and amikacin were used to treat the patient. Penicillinase-resistant penicillins or first-generation cephalosporin are the good initial choices to effectively control S. aureus, along with clindamycin or a similar medication to control possible anaerobic infections. The present patient received a seven-day treatment. The shortest duration reported in treating $S$. aureus parotitis in the absence of septice- mia was seven days (9). Neonatal suppurative parotitis is more prevalent among boys $(2,10)$. In the current report, patient was girl. Medical treatments are sufficient in most of the cases (11). Antimicrobial treatment lead to clinical improvement in majority of the patients in the study by Spiegel et al. (2). Complications such as facial palsy, mediastinitis, salivary fistula and extension to external ear are infrequent due prompt antibiotic therapy (12). Surgical treatment is used when there is intraparotid abscess (2). The present patient showed improvement without surgical intervention observed in most of the reports. The present patient fulfilled the diagnostic criteria. Parotid swelling, purulent exudates from the Stensen's duct and growth of the pathogenic bacteria in the parotid plus culture were the diagnostic criteria (2). In conclusion, acute suppurative parotitis should be suspected in infants presenting facial swelling, redness of pre-auricular region, and fever. Treatment with appropriate intravenous antibiotics is recommended.

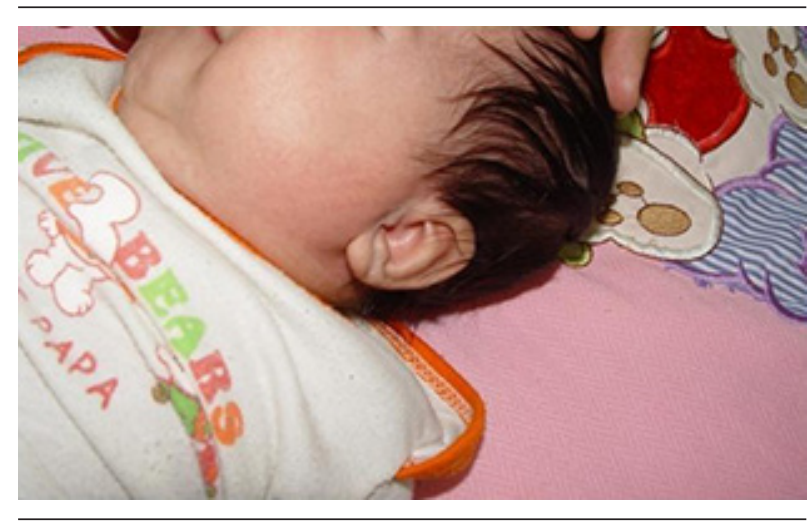

Figure 1. Left Facial Swelling

\section{Acknowledgements}

Authors acknowledge their gratitude to the parents of the child for their cooperation.

\section{Authors' Contributions}

Seyed Mohammad Hassan Aletayeb: Diagnosis and treatment of patient, revision of manuscript; Ashraf Sepehran: Diagnosis and treatment; Hazhir Javaherizadeh: Preparation of manuscript, searching the literatures, responsible for final revision.

\section{References}

1. Leake D, Leake R. Neonatal suppurative parotitis. Pediatrics. 1970;46(2):202-7.

2. Spiegel R, Miron D, Sakran W, Horovitz Y. Acute neonatal suppurative parotitis: case reports and review. Pediatr Infect Dis $J$. 2004;23(1):76-8.

3. Decembrino L, Ruffinazzi G, Russo F, Manzoni P, Stronati M Monolateral suppurative parotitis in a neonate and review of literature. Int J Pediatr Otorhinolaryngol. 2012;76(7):930-3.

4. Schwab J, Baroody F. Neonatal suppurative parotitis: a case report. Clin Pediatr (Phila). 2003;42(6):565-6.

5. Ismail EA, Seoudi TM, Al-Amir M, Al-Esnawy AA. Neonatal suppu- 


\section{Aletayeb SMH et al.}

rative parotitis over the last 4 decades: report of three new cases and review. Pediatr Int. 2013;55(1):60-4.

6. Singh K. Bilateral parotid abscess in a neonate. Indian Pediatr 2006;43(11):1009-10.

7. Chevalier J, Jadcherla SR. Parotid swelling in a premature neonate. Am J Perinatol. 2002;19(8):435-8.

8. Donovan ST, Rohman GT, Selph JP, Rajan R, Stocks RM, Thompson JW. Methicillin-resistant Staphylococcus aureus as a cause of neonatal suppurative parotitis: a report of two cases and review of the literature. Ear Nose Throat J. 2013;92(6):269-71.
9. Todoroki Y, Tsukahara H, Kawatani M, Ohshima Y, Shukunami K, Kotsuji F, et al. Neonatal suppurative parotitis possibly associated with congenital cytomegalovirus infection and maternal methyldopa administration. Pediatr Int. 2006;48(2):185-6.

10. Salaria M, Poddar B, Parmar V. Neonatal parotitis. Indian J Pediatr. 2001;68(3):283.

11. Khan SU, O'Sullivan PG, McKiernan J. Acute suppurative neonatal parotitis: Case report. Ear Nose Throat J. 2010;89(2):90-1.

12. Mohyud-Din M, Haider S, Hameed A. Bilateral suppurative parotitis in a newborn. J Coll Physicians Surg Pak. 2006;16(4):301-2. 